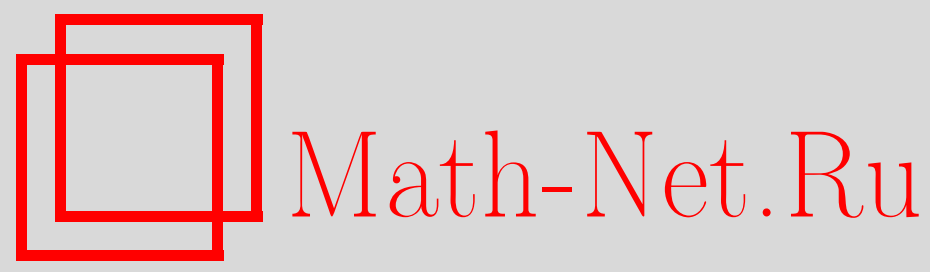

А. И. Козко, Аналоги неравенств Джексона-Никольского для тригонометрических полиномов в пространствах с несимметричной нормой, Матем. заметки, 1997, том 61, выпуск 5, 687-699

DOI: https://doi.org/10.4213/mzm1550

Использование Общероссийского математического портала Math-Net.Ru подразумевает, что вы прочитали и согласны с пользовательским соглашением http://www . mathnet.ru/rus/agreement

Параметры загрузки:

IP: 3.80 .181 .102

26 апреля 2023 г., 08:29:40

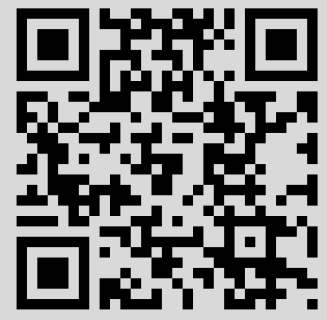




\section{АНАЛОГИ НЕРАВЕНСТВ ДЖЕКСОНА-НИКОЛЬСКОГО ДЛЯ ТРИГОНОМЕТРИЧЕСКИХ ПОЛИНОМОВ В ПРОСТРАНСТВАХ С НЕСИММЕТРИЧНОЙ НОРМОЙ}

\section{А. И. Козко}

Исследуется величина

$$
\sup _{\substack{t_{n} \in T_{n} \\ t_{n} \not \equiv 0}} \frac{\left\|t_{n}\right\|_{q_{1}, q_{2}}}{\left\|t_{n}\right\|_{p_{1}, p_{2}}}
$$

где $\|\cdot\|_{p_{1}, p_{2}}-$ несимметричная норма. Вычисляется ее порядок. Для оценок снизу приводятся новые полиномы, свойства которых детально изучаются. Данная оценка в случае $p_{1}=p_{2}, q_{1}=q_{2}$ представляет собой хорошо известное неравенство Джексона-Никольского.

Библиограбфия: 5 названий.

1. В работе устанавливается неравенство типа Джексона-Никольского для тригонометрических полиномов в пространствах с несимметричной нормой.

Введем обозначения: $L_{p}[0,2 \pi](1 \leqslant p<\infty)$ - пространство суммируемых по Лебегу на $[0,2 \pi]$ в $p$-й степени функций $x(\cdot)$ с нормой

$$
\|x\|_{p}=\left\{\int_{0}^{2 \pi}|x(t)|^{p} d t\right\}^{1 / p},
$$

$L_{\infty}[0,2 \pi]-$ пространство измеримых и существенно ограниченных на $[0,2 \pi]$ функций $x(\cdot)$, в котором норма задается равенством

$$
\|x\|_{\infty}=\sup _{0 \leqslant t \leqslant 2 \pi} \operatorname{vrai}|x(t)| .
$$

Пусть $n$ - натуральное число, $T_{n}$ - множество всех тригонометрических полиномов степени не вьше $n$, т.е. $t_{n} \in T_{n}$ означает, что

$$
t_{n}(x)=\frac{a_{0}}{2}+\sum_{k=1}^{n}\left\{a_{k} \cos k x+b_{k} \sin k x\right\},
$$

где $a_{k}$ и $b_{k}$ - постоянные коэффициенты, вообще говоря, комплексные. Для тригонометрических полиномов степени $n$ (см. $[1$, с. 133]) С. М. Никольский установил неравенство

$$
\left\|t_{n}\right\|_{q} \leqslant 3 n^{1 / p-1 / q}\left\|t_{n}\right\|_{p}, \quad 1 \leqslant p \leqslant q \leqslant \infty
$$


и применил его для получения теорем вложения. В случае $q=\infty$ соответствующее неравенство доказал Джексон (см. [2, с. 891]). Неравенство (1.1) в смыслепорядка точное, что проверяется на тригонометрическом ядре Фейера

$$
F_{n}(x)=\frac{1}{2}+\sum_{k=1}^{n}\left(1-\frac{k}{n+1}\right) \cos k x=\frac{\sin ^{2} \frac{n+1}{2} x}{2(n+1) \sin ^{2} \frac{x}{2}} .
$$

Здесь и далее будем рассматривать только действительнозначные функции. Положим $f^{+}(t)=\max \{f(t), 0\}, f^{-}(t)=\max \{-f(t), 0\}$. Пусть $\|f\|_{p, q}=\left\|f^{+}\right\|_{p}+\left\|f^{-}\right\|_{q}$.

Будем писать $f \in L_{p, q}[0,2 \pi]$, если $f^{+} \in L_{p}[0,2 \pi]$ и $f^{-} \in L_{q}[0,2 \pi]$. Несимметричная норма $\|\cdot\|_{p, q}: L_{p, q}[0,2 \pi] \rightarrow \mathbb{R}_{+}$удовлетворяет следующим условиям:

1) $\|f\|_{p, q}=0 \Longleftrightarrow f=0$ почти всюду на $[0,2 \pi]$;

2) $\|f+g\|_{p, q} \leqslant\|f\|_{p, q}+\|g\|_{p, q}, f, g \in L_{p, q}[0,2 \pi]$;

3) $\|\alpha f\|_{p, q}=\alpha\|f\|_{p, q}, f \in L_{p, q}[0,2 \pi], \alpha \in \mathbb{R}_{+}$.

Пусть $f$ и $g$ - функции натурального параметра. Будем писать $f \asymp g$, если существуют константы $C_{1}, C_{2}>0$, не зависящие от $n$, такие, что $C_{1}|f(n)| \leqslant|g(n)| \leqslant C_{2}|f(n)|$, $n \in \mathbb{N}$.

В настоящей работе устанавливаются следующие результаты.

Tеорема 1. Пусть $1 \leqslant p \leqslant \infty, n \in \mathbb{N}$. Тогдa

$$
\sup _{\substack{t_{n} \in T_{n} \\ t_{n} \not \equiv 0}} \frac{\left\|t_{n}\right\|_{1, \infty}}{\left\|t_{n}\right\|_{1, p}} \asymp n^{3 /(2 p+1)}
$$

TEорема 2. Пусть $p_{1}, p_{2}, q_{1}, q_{2} \in[1, \infty], n \in \mathbb{N}$. Тољдa

$$
\sup _{\substack{t_{n} \in T_{n} \\ t_{n} \not \equiv 0}} \frac{\left\|t_{n}\right\|_{q_{1}, q_{2}}}{\left\|t_{n}\right\|_{p_{1}, p_{2}}} \asymp n^{\psi\left(p_{1}, p_{2}, q_{1}, q_{2}\right)},
$$

әде

$$
\psi\left(p_{1}, p_{2}, q_{1}, q_{2}\right)= \begin{cases}\max \left\{\left(\frac{1}{p_{1}}-\frac{1}{q_{1}}\right)_{+},\left(\frac{2+1 / p_{1}}{2+1 / p_{2}}\right)\left(\frac{1}{p_{2}}-\frac{1}{q_{2}}\right)_{+}\right\}, & p_{1} \leqslant p_{2}, \\ \max \left\{\left(\frac{1}{p_{1}}-\frac{1}{q_{1}}\right)_{+}\left(\frac{2+1 / p_{2}}{2+1 / p_{1}}\right),\left(\frac{1}{p_{2}}-\frac{1}{q_{2}}\right)_{+}\right\}, \quad p_{1} \geqslant p_{2}\end{cases}
$$

$u \alpha_{+}=\max \{0, \alpha\}$.

Теорема 2 является простым следствием теоремы 1. Оценка сверху в (1.3) будет получена с помощью функции сравнения вида $a \cos n x+b$, а оценка снизу может быть получена на полиномах вида

$$
S t_{n}(\delta, t)=\frac{F_{n}(t)\left(\cos t-\cos \left(\frac{2 \pi}{n+1}-\delta\right)\right)\left(\cos t-\cos \left(\frac{2 \pi}{n+1}+\delta\right)\right)}{\left(\cos t-\cos \frac{2 \pi}{n+1}\right)^{2}},
$$

которые были предложены автору С. Б. Стечкиным. Будет показано, что оценка снизу достигается также на полиномах

$$
T_{n}(\delta, t)=\frac{F_{n+1}(t)(\cos \delta-\cos t)}{\left(\cos t-\cos \frac{2 \pi}{n+2}\right)^{2}(n+2)^{2}} .
$$

Подробнее об экстремальных полиномах будет сказано ниже. 
2. В этом пункте докажем, что если $n \in \mathbb{N}, t_{n} \in T_{n}, p \in[1,+\infty]$, то

$$
\left\|t_{n}\right\|_{1, \infty} \leqslant e^{1 / e} n^{3 /(2 p+1)}\left\|t_{n}\right\|_{1, p}
$$

Положим $M^{+}=\left\|t_{n}^{+}\right\|_{\infty}, M^{-}=\left\|t_{n}^{-}\right\|_{\infty}, M=\left\|t_{n}\right\|_{\infty}$. Верна следующая лемма.

Лемма 1. Пусть $n \in \mathbb{N} u t_{n} \in T_{n}, x_{0} \in[0,2 \pi]$ mаковы, ито $M^{-}=-t_{n}\left(x_{0}\right) \neq 0$. Положим

$$
\varphi(x)=-\frac{M^{+}+M^{-}}{2} \cos n x+\frac{M^{+}-M^{-}}{2},
$$

тогда для любого $x \in[-\pi /(2 n), \pi /(2 n)]$ имеем $t_{n}\left(x+x_{0}\right) \leqslant \varphi(x)$.

ДокАзАтЕльство. Данная лемма вытекает из работы Стечкина (см. [3, с. 22]).

Заметим, что справедливы следующие элементарные неравенства:

$$
\begin{gathered}
\arccos (1-x) \geqslant \sqrt{x} \quad \text { при } 0 \leqslant x \leqslant 2 ; \\
a^{\alpha} b^{\beta} \leqslant(a+b), \quad a, b, \alpha, \beta \geqslant 0, \quad \alpha+\beta=1 .
\end{gathered}
$$

Лемма 2. Пусть $n \in \mathbb{N}, t_{n} \in T_{n}, p \in[1,+\infty]$.

a) $E с л и M^{-}=M, m o$

$$
M^{-} \leqslant\left(\frac{p+1}{\pi}\right)^{1 / p} n^{1 / p}\left\|t_{n}^{-}\right\|_{p}
$$

b)

$$
M^{-} \leqslant\left(\frac{p+1}{2}\right)^{2 /(2 p+1)} n^{2 /(2 p+1)}\left\|t_{n}^{-}\right\|_{p}^{2 p /(2 p+1)}\left(M^{+}\right)^{1 /(2 p+1)} .
$$

ДоКАЗАТЕЛЬСТВО. При $p=\infty$ или $M^{-}=0$ утверждения (2.2) и (2.3) очевидны. Без ограничения общности положим $x_{0}=0$. Пусть $M^{-}=M \geqslant M^{+}$. Тогда для любых $x \in[-\pi /(2 n), \pi /(2 n)]$ имеем $\varphi(x) \leqslant 0$, и согласно лемме 1

$$
t_{n}(x) \leqslant \varphi(x) \leqslant M^{-}\left(\frac{2 n}{\pi}|x|-1\right) \leqslant 0, \quad|x| \leqslant \frac{\pi}{2 n} .
$$

Следовательно,

$$
\left\|t_{n}^{-}\right\|_{p}^{p} \geqslant 2 \int_{0}^{\pi /(2 n)}\left(M^{-}\left(-\frac{2 n}{\pi} x+1\right)\right)^{p} d x=\frac{2}{p+1} \cdot \frac{\pi}{2 n}\left(M^{-}\right)^{p}=\frac{\pi}{(p+1) n}\left(M^{-}\right)^{p}
$$

откуда вытекает оценка (2.2).

Пусть $M^{-}<M^{+}=M$. Тогда $\varphi(x)$ имеет нули и $\varphi(0) \neq 0$. Пусть $y_{1}-$ наименьший положительньй нуль функции $\varphi(x)$. Имеем

$$
y_{1}=\frac{\arccos \left(1-2 M^{-} /\left(M^{+}+M^{-}\right)\right)}{n}, \quad y_{1} \in\left[0, \frac{\pi}{2 n}\right] \text {. }
$$


Лемма 1 показьвает, что

$$
t_{n}(x) \leqslant \varphi(x) \leqslant M^{-}\left(\frac{|x|}{y_{1}}-1\right) \leqslant 0, \quad|x| \leqslant y_{1} .
$$

Следовательно,

$$
\begin{aligned}
\left\|t_{n}^{-}\right\|_{p}^{p} & \geqslant 2 \int_{0}^{y_{1}}\left(-\frac{M^{-}}{y_{1}} x+M^{-}\right)^{p} d x=\frac{2}{p+1} y_{1}\left(M^{-}\right)^{p} \\
& =\frac{2}{p+1} \cdot \frac{\left(M^{-}\right)^{p}}{n} \arccos \left(1-\frac{2 M^{-}}{M^{+}+M^{-}}\right) \geqslant \frac{2}{p+1} \cdot \frac{\left(M^{-}\right)^{p}}{n} \sqrt{\frac{2 M^{-}}{M^{+}+M^{-}}} \\
& \geqslant \frac{2}{p+1} \cdot \frac{1}{n}\left(M^{-}\right)^{p} \sqrt{\frac{M^{-}}{M^{+}}} .
\end{aligned}
$$

Отсюда следует (2.3), и лемма доказана.

Заметим, что для любого $p \in[1,+\infty]$ имеем

$$
\left(\frac{p+1}{2}\right)^{2 /(2 p+1)} \leqslant p^{2 /(2 p+1)} \leqslant p^{1 / p} \leqslant e^{1 / e}, \quad\left(\frac{p+1}{\pi}\right)^{1 / p} \leqslant p^{1 / p} \leqslant e^{1 / e}
$$

тем самым, показано, что константы в $(2.2),(2.3)$ ограничены.

УТВЕРЖДЕНИЕ 1. Пусть $n \in \mathbb{N}, t_{n} \in T_{n}$, тогда

$$
\left\|t_{n}\right\|_{1,+\infty} \leqslant e^{1 / e} n^{3 /(2 p+1)}\left\|t_{n}\right\|_{1, p}
$$

ДоКАЗАТЕЛЬСтво. Заметим, что достаточно доказать неравенство

$$
M^{-} \leqslant e^{1 / e} n^{3 /(2 p+1)}\left\|t_{n}\right\|_{1, p} \quad \forall p \in[1,+\infty]
$$

Если $M^{-}=M$, то оценка (2.5) вытекает немедленно из (2.2)

$$
M^{-} \leqslant e^{1 / e} n^{1 / p}\left\|t_{n}^{-}\right\|_{p} \leqslant e^{1 / e} n^{3 /(2 p+1)}\left\|t_{n}\right\|_{1, p}, \quad p \in[1,+\infty] .
$$

В случае, если $M^{-}<M^{+}=M$, имеем из $(2.3)\left(M^{+} \leqslant n\left\|t_{n}^{+}\right\|_{1}\right.$ по лемме 2, случай а) $)$

$$
\begin{aligned}
M^{-} & \leqslant e^{1 / e} n^{2 /(2 p+1)}\left\|t_{n}^{-}\right\|_{p}^{2 p /(2 p+1)}\left(M^{+}\right)^{1 /(2 p+1)} \\
& \leqslant e^{1 / e} n^{2 /(2 p+1)} n^{1 /(2 p+1)}\left\|t_{n}^{-}\right\|_{p}^{2 p /(2 p+2)}\left\|t_{n}^{+}\right\|_{1}^{1 /(2 p+1)} \\
& \leqslant e^{1 / e} n^{3 /(2 p+1)}\left\|t_{n}^{-}\right\|_{p}^{2 p /(2 p+1)}\left\|t_{n}^{+}\right\|_{1}^{1 /(2 p+1)} \leqslant e^{1 / e} n^{3 /(2 p+1)}\left\|t_{n}\right\|_{1, p}
\end{aligned}
$$

в последнем неравенстве мы воспользовались оценкой $(2.1)$, взяв в ней $a=\left\|t_{n}^{-}\right\|_{p}$, $b=\left\|t_{n}\right\|_{1}$. Таким образом, оценка (2.5) доказана. Утверждение доказано. 
3. В этом пункте покажем, что неравенство (2.4), полученное в утверждении 1 , является точньм по порядку, откуда будет следовать, что верна оценка (1.3). Для этого нам потребуются ряд вспомогательных утверждений о свойствах полинома

$$
T_{n}(\delta, t)=\frac{F_{n+1}(t)(\cos \delta-\cos t)}{\left(\cos t-\cos \frac{2 \pi}{n+2}\right)^{2}(n+2)^{2}}, \quad t \in[0,2 \pi], \quad \delta \in\left[0, \frac{1}{2 n}\right]
$$

где $F_{n+1}(\cdot)$ - ядро Фейера (1.2).

ПРЕДЛОЖЕНИЕ 1. Справедливы следующие утверждения:

a) npu всеx $t \in(-\infty,+\infty)$

$$
\sin ^{2} \frac{n+2}{2} t \leqslant\left\{(n+2) \sin \left(\frac{t}{2}-\frac{\pi}{n+2}\right)\right\}^{2}
$$

b) при всех $t \in[\pi /(n+2), 3 \pi /(n+2)]$

$$
\sin ^{2} \frac{n+2}{2} t \geqslant\left(\frac{2}{\pi}\right)^{2}(n+2)^{2}\left(\frac{t}{2}-\frac{\pi}{n+2}\right)^{2} ;
$$

c) при всех $t \in[0,3 \pi /(n+2)]$

$$
\sin \left(\frac{t}{2}+\frac{\pi}{n+2}\right) \geqslant \frac{1}{n+2}
$$

d) $n p$ и всех $t \in[3 \pi /(2(n+2)), 5 \pi /(2(n+2))]$

$$
\sin ^{2}\left(\frac{t}{2}+\frac{\pi}{n+2}\right) \leqslant \frac{25}{4} \pi^{2}\left(\frac{1}{n+2}\right)^{2} .
$$

ДокАЗАТЕЛЬСтво. Имеют место следующие, важные сами по себе, неравенства:

$$
\begin{aligned}
|\sin n t| & \leqslant n|\sin t| & \text { при всех } t & \in(-\infty, \infty), \\
\sin t & \geqslant \frac{2}{\pi} t & \text { при всех } t & \in\left[0, \frac{\pi}{2}\right], \\
|\sin t| & \leqslant t & \text { при всех } t & \geqslant 0,
\end{aligned}
$$

(cм. [4, c. 101]).

Случаи а), b) есть следствие (3.6), (3.7), так как

$$
\sin ^{2} \frac{n+2}{2} t=\sin ^{2}(n+2)\left(\frac{t}{2}-\frac{\pi}{n+2}\right)
$$

При $n=1$ непосредственной проверкой можно убедиться, что для $t \in[0,3 \pi /(n+2)]$ имеем

$$
\sin \left(\frac{t}{2}+\frac{\pi}{n+2}\right) \geqslant \sin \frac{5 \pi}{2(n+2)} \geqslant \sin \frac{\pi}{2(n+2)} \geqslant \frac{1}{n+2} .
$$


При $n \geqslant 2$

$$
\sin \left(\frac{t}{2}+\frac{\pi}{n+2}\right) \geqslant \sin \frac{\pi}{n+2} \geqslant \frac{2}{\pi} \cdot \frac{\pi}{n+2}=\frac{2}{n+2} .
$$

Итак, при $t \in[0,3 \pi /(n+2)]$ получили утверждение $c)$.

Так как $t \in[3 \pi /(2(n+2)), 5 \pi /(2(n+2))]$, то непосредственно можно убедиться, что при $n=1,2$

$$
\sin ^{2}\left(\frac{t}{2}+\frac{\pi}{n+2}\right) \leqslant \sin ^{2} \frac{7 \pi}{4(n+2)} \leqslant \frac{49}{(n+2)^{2}}\left(\frac{\pi}{4}\right)^{2} .
$$

При $n \geqslant 3$

$$
\sin ^{2}\left(\frac{t}{2}+\frac{\pi}{n+2}\right) \leqslant \sin ^{2} \frac{9 \pi}{4(n+2)} \leqslant \frac{81}{16} \pi^{2} \frac{1}{(n+2)^{2}} .
$$

Итак, при $t \in[3 \pi /(2(n+2)), 5 \pi /(2(n+2))]$ утверждение $\mathrm{d})$ доказано.

Предложение доказано.

Полином $T_{n}(\delta, t)$ может быть преобразован к виду

$$
T_{n}(\delta, t)=\frac{\sin ^{2} \frac{n+2}{2} t \sin \frac{t-\delta}{2} \sin \frac{t+\delta}{2}}{(n+2)^{3} \sin ^{2} \frac{t}{2} \sin ^{2}\left(\frac{t}{2}+\frac{\pi}{n+2}\right) \sin ^{2}\left(\frac{t}{2}-\frac{\pi}{n+2}\right)},
$$

откуда немедленно следует, что

$$
\begin{gathered}
T_{n}(\delta, t) \leqslant 0 \quad \text { при } t \in[-\delta, \delta], \\
T_{n}(\delta, t) \geqslant 0 \quad \text { при } \quad t \in[-\pi,-\delta] \cup[\delta, \pi] .
\end{gathered}
$$

Это следует также из формулы (3.1).

ЛЕмма 3. Для полинома $T_{n}(\delta, t)$ при $\delta \in(0, \pi /(2 n))$ справедливо

$$
M^{+}=\left\|T_{n}^{+}(\delta, \cdot)\right\|_{\infty}=\left\|T_{n}(\delta, \cdot)\right\|_{\infty}=M .
$$

ДокАЗАТЕЛЬСтво. Предположим противное, $M^{+} \neq M$, т.е. $M=M^{-}$. Пусть $x_{0}$ есть точка, в которой $T_{n}\left(\delta, x_{0}\right)=-M^{-}$. Тогда по лемме Стечкина (см. [3, с. 22])

$$
\left|T_{n}\left(\delta, x+x_{0}\right)\right| \geqslant\left|M^{-} \cos n x\right|, \quad x \in\left[-\frac{\pi}{2 n}, \frac{\pi}{2 n}\right] .
$$

Откуда

$$
\mu\left\{t \in[-\pi, \pi] \mid T_{n}(\delta, x) \leqslant 0\right\} \geqslant \frac{\pi}{n} .
$$

Но, с другой стороны, из явного вида $T_{n}(\delta, t)$ (формула (3.1)) получаем

$$
\mu\left\{t \in[-\pi, \pi] \mid T_{n}(\delta, t) \leqslant 0\right\}=2 \delta<\frac{\pi}{n},
$$

где использовалось, что $\delta<\pi /(2 n)$. Полученное соотношение противоречит (3.10).

Следовательно, допущение $M^{-}=M$ было неверным. Лемма доказана. 
Лемма 4. При $\delta \in(0,1 /(2 n))$ верны следующие оценки:

$$
\begin{gathered}
\left|T_{n}(\delta, t)\right| \leqslant C_{1} F_{n+1}(t)=C_{1} \frac{\sin ^{2} \frac{n+2}{2} t}{2(n+2) \sin ^{2} \frac{t}{2}} \quad \forall t \in\left[\frac{3 \pi}{n+2}, \pi\right] \\
\left|T_{n}(\delta, t)\right| \leqslant C_{2} n \quad \forall t \in\left[\delta, \frac{3 \pi}{n+2}\right]
\end{gathered}
$$

где $C_{1}, C_{2}>0$ - константы, не зависящие от $n$ и $\delta$.

ДокАЗАТЕЛЬСтво. Так как при $n \leqslant 4$ лемма тривиальна, рассмотрим случай когда $n \geqslant 4$. В силу (3.9) для обоснования (3.11) достаточно доказать, что

$$
R_{n}(\delta, t)=\frac{2 \sin \frac{t-\delta}{2} \sin \frac{t+\delta}{2}}{(n+2)^{2} \sin ^{2}\left(\frac{t}{2}+\frac{\pi}{n+2}\right) \sin ^{2}\left(\frac{t}{2}-\frac{\pi}{n+2}\right)} \leqslant C_{1}
$$

при $t \in[3 \pi /(n+2), \pi]$. В случае $t \in[3 \pi /(n+2), \pi-2 \pi /(n+2)]$, согласно $(3.7),(3.8)$ имеем

$$
\begin{aligned}
R_{n}(\delta, t) & \leqslant \frac{2}{(n+2)^{2}} \frac{\frac{t^{2}-\delta^{2}}{4}}{\left(\frac{2}{\pi}\right)^{4}\left(\frac{t}{2}+\frac{\pi}{n+2}\right)^{2}\left(\frac{t}{2}-\frac{\pi}{n+2}\right)^{2}} \leqslant \frac{2}{(n+2)^{2}} \frac{\frac{t^{2}}{4}}{\left(\frac{2}{\pi}\right)^{4} \frac{t^{2}}{4}\left(\frac{t}{2}-\frac{\pi}{n+2}\right)^{2}} \\
& \leqslant \frac{2}{(n+2)^{2}} \frac{1}{\left(\frac{2}{\pi}\right)^{4}\left(\frac{\pi}{2(n+2)}\right)^{2}} \leqslant \frac{\pi^{2}}{2} .
\end{aligned}
$$

В случае $t \in[\pi-2 \pi /(n+2), \pi]$ при $n \geqslant 4$, вьполняется неравенство

$$
\sin \left(\frac{t}{2} \pm \frac{\pi}{n+2}\right) \geqslant \frac{1}{2}
$$

и потому

$$
R_{n}(\delta, t) \leqslant \frac{2}{\frac{1}{2} \cdot \frac{1}{2}}=8
$$

Тем самым, (3.11) доказано.

Докажем (3.12). Используя (3.7), (3.8), (3.2), (3.4), имеем

$$
\begin{aligned}
& \frac{\sin ^{2} \frac{n+2}{2} t \sin \frac{t-\delta}{2} \sin \frac{t+\delta}{2}}{(n+2)^{3} \sin ^{2} \frac{t}{2} \sin ^{2}\left(\frac{t}{2}+\frac{\pi}{n+2}\right) \sin ^{2}\left(\frac{t}{2}-\frac{\pi}{n+2}\right)} \\
& \leqslant \frac{(n+2)^{2} \sin ^{2}\left(\frac{t}{2}-\frac{\pi}{n+2}\right) \cdot \frac{t^{2}-\delta^{2}}{4}}{(n+2)^{3} \sin ^{2} \frac{t}{2} \sin ^{2}\left(\frac{t}{2}+\frac{\pi}{n+2}\right) \sin ^{2}\left(\frac{t}{2}-\frac{\pi}{n+2}\right)} \\
& \leqslant \frac{\left(\frac{t}{2}\right)^{2}}{(n+2)\left(\frac{2}{\pi}\right)^{2}\left(\frac{t}{2}\right)^{2} \sin ^{2}\left(\frac{t}{2}+\frac{\pi}{n+2}\right)} \\
& \leqslant \frac{1}{(n+2)\left(\frac{2}{\pi}\right)^{2} \frac{1}{(n+2)^{2}}}=C(n+2) \leqslant C_{2} n .
\end{aligned}
$$

Лемма доказана. 
Лемма 5. Пусть $n \in \mathbb{N}, \delta \in(0,1 /(2 n))$. Тогда

$$
\left|T_{n}(\delta, t)\right| \geqslant C n \quad \forall t \in\left[\frac{3 \pi}{2(n+2)}, \frac{5 \pi}{2(n+2)}\right] .
$$

ДоказАТЕЛЬСТво. Согласно (3.3)-(3.5), (3.8) имеем

$$
\left|T_{n}(\delta, t)\right| \geqslant \frac{1}{(n+2)^{3}} \frac{\left(\frac{2}{\pi}\right)^{2}(n+2)^{2}\left(\frac{\pi}{2(n+2)}\right)^{2}\left|\frac{3 \pi}{2(n+2)}-\delta\right| \frac{3 \pi}{2(n+2)}}{\left(\frac{5 \pi}{4(n+2)}\right)^{2} \frac{25}{4} \pi^{2} \frac{1}{(n+2)^{2}}\left(\frac{\pi}{2}\right)^{2} \frac{1}{(n+2)^{2}}} \geqslant C n,
$$

так как

$$
\left|\frac{3 \pi}{2(n+2)}-\delta\right| \geqslant\left|\frac{3 \pi}{2(n+2)}-\frac{1}{2 n}\right|=\left|\frac{(3 \pi-1) n-2}{2 n(n+2)}\right| \geqslant\left|\frac{8 n-2}{2 n(n+2)}\right| \geqslant \frac{1}{n+2} .
$$

Здесь использовано то, что $\delta<1 /(2 n)$. Лемма доказана.

Лемма 6. Пусть $n \in \mathbb{N}, p \in[1,+\infty], \delta \in(0,1 /(2 n))$. Тогда

$$
\left\|T_{n}^{+}(\delta, \cdot)\right\|_{p} \asymp n^{1-1 / p} .
$$

с константами, зависящими только от $р$.

ДоказАтельство. а) Оценка $\left\|T_{n}^{+}(\delta, \cdot)\right\|_{p} \leqslant C n^{1-1 / p}$ для $p=\infty$ следует из леммы 4 . Для $p \in[1, \infty)$ имеем (см. [5, с. 245])

$$
\left\|F_{n+1}(\cdot)\right\|_{p}^{p} \asymp n^{p-1} \quad \forall p \in[1, \infty],
$$

и потому

$$
\left\|T_{n}^{+}(\delta, \cdot)\right\|_{p}^{p} \leqslant 2\left\{\int_{\delta}^{3 \pi /(n+2)}\left|T_{n}(\delta, t)\right|^{p} d t+C_{1}\left\|F_{n+1}(\cdot)\right\|_{p}^{p}\right\} \leqslant C n^{p-1},
$$

где опять мы воспользовались леммой 4 , но уже оценкой (3.12) и тем, что

$$
\left(\frac{3 \pi}{2(n+2)}\right)-\delta \leqslant \frac{3 \pi}{n+2}
$$

при $0<\delta<1 /(2 n)$.

b) При $p=\infty$ нужная оценка снизу для $\left\|T_{n}^{+}(\delta, \cdot)\right\|_{p}$ сразу следует из леммы 5 ; если же $1 \leqslant p<\infty$, то вновь в силу леммы 5

$$
\left\|T_{n}^{+}(\delta, \cdot)\right\|_{p} \geqslant\left\{\int_{3 \pi /(2(n+2))}^{5 \pi /(2(n+2))}\left|T_{n}^{+}(\delta, t)\right|^{p} d t\right\}^{1 / p} \geqslant C_{1} n\left(\frac{\pi}{n+2}\right)^{1 / p} \geqslant C n^{1-1 / p}
$$

Лемма доказана. 
ЛЕмма 7. Для полинома $T_{n}(\delta, t)$, определенного формулой (1.4),

$$
M^{-}=\left\|T_{n}^{-}(\delta, \cdot)\right\|_{\infty} \geqslant C \delta^{2} n^{3}
$$

при $0<\delta<1 /(2 n)$, әде $C>0$ - константа, не зависящая от $n$ и $\delta$.

ДокАЗАТЕЛЬСТво. Имеем

$\left|T_{n}(\delta, 0)\right|=\lim _{t \rightarrow 0} \frac{\sin ^{2} \frac{n+2}{2} t\left|\sin \frac{t-\delta}{2}\right| \sin \frac{t+\delta}{2}}{(n+2)^{3} \sin ^{2} \frac{t}{2} \sin ^{2}\left(\frac{t}{2}+\frac{\pi}{n+2}\right) \sin ^{2}\left(\frac{t}{2}-\frac{\pi}{n+2}\right)}=\frac{1}{n+2} \frac{\sin ^{2} \frac{\delta}{2}}{\sin ^{4} \frac{\pi}{n+2}} \geqslant C \delta^{2} n^{3}$.

Лемма доказана.

Рассмотрим полином $t_{n} \in T_{n}$ такой, что $M^{-} \neq 0, M^{+} \neq 0$. Тогда существует отрезок $\left[x_{1}, x_{2}\right]$ такой, что

1) $\exists x^{-} \in\left[x_{1}, x_{2}\right]-t_{n}\left(x^{-}\right)=M^{-}$;

2) $\forall x \in\left[x_{1}, x_{2}\right] t_{n}(x) \leqslant 0$

3) $t_{n}\left(x_{1}\right)=t_{n}\left(x_{2}\right)=0$.

Положим $\delta^{*}=\min \left\{\left|x_{1}-x^{-}\right|,\left|x_{2}-x^{-}\right|\right\}$.

Предположим, что этот минимум равен $\left|x_{1}-x^{-}\right|$. Разложим в ряд Тейлора в точке $x^{-}$полином $t_{n} \in T_{n}$

$$
t_{n}\left(x^{-}+\delta^{*}\right)=t_{n}\left(x^{-}\right)+\frac{t_{n}^{(2)}\left(x^{-}\right)}{2 !}\left(\delta^{*}\right)^{2}+\cdots+\frac{t_{n}^{(k)}\left(x^{-}\right)}{k !}\left(\delta^{*}\right)^{k}+\cdots,
$$

здесь $t_{n}^{\prime}\left(x^{-}\right)=0$, так как $x^{-}$- точка, в которой полином достигает своего минимума.

Предположим, что $\delta^{*} \in(0,1 /(2 n))$, тогда в силу неравенства Бернштейна $\left|t_{n}^{(k)}\left(x^{-}\right)\right| \leqslant$ $n^{k}\left\|t_{n}\right\|_{\infty}=n^{k} M$ получаем

$$
\begin{aligned}
M^{-} & =\left|\frac{t_{n}^{(2)}\left(x^{-}\right)}{2 !}\left(\delta^{*}\right)^{2}+\cdots+\frac{t_{n}^{(k)}\left(x^{-}\right)}{k !}\left(\delta^{*}\right)^{k}+\cdots\right| \\
& \leqslant n^{2}\left(\delta^{*}\right)^{2} M+\cdots+n^{k}\left(\delta^{*}\right)^{k} M+\cdots=M \frac{n^{2}\left(\delta^{*}\right)^{2}}{1-n \delta^{*}} \leqslant 2 M n^{2}\left(\delta^{*}\right)^{2},
\end{aligned}
$$

где последнее неравенство написано с учетом того, что сумма образует геометрическую прогрессию с $q=n \delta^{*}<1 / 2$.

Лемма 8. Пусть $n \in \mathbb{N}, p \in[1,+\infty], \delta \in(0,1 /(2 n))$, погда для полинома $T_{n}(\delta, t)$ имеем

$$
M^{-} \asymp n^{3} \delta^{2} .
$$

ДокАЗАТЕЛЬСТво. Пусть $x_{0} \in[-\delta, \delta]$ такая,что $\left|T_{n}\left(\delta, x_{0}\right)\right|=M^{-}$и

$$
\delta^{*}=\min \left\{\left|\delta-x_{0}\right|,\left|x_{0}+\delta\right|\right\} \leqslant \delta,
$$

используя (3.14) и (3.13), а также, учитывая результат леммы 3 , что $M^{+}=M$, имеем $M^{-} \leqslant 2 n^{2} \delta^{2} M=2 n^{2} \delta^{2} M^{+} \leqslant C n^{3} \delta^{2}$. Оценка снизу следует из леммы 7 . Лемма доказана. 
Лемма 9. Пусть $n \in \mathbb{N}, p \in[1,+\infty], \delta \in(0,1 /(2 n))$. Тогда

$$
\left\|T_{n}^{-}(\delta, \cdot)\right\|_{p} \asymp n^{3} \delta^{2+1 / p}
$$

с константами, зависящими только от р.

ДоказАТЕльство. Так как при $p=\infty$ уже все доказано (см. (3.15)), то достаточно доказать утверждение леммы для случая $1 \leqslant p<\infty$

$$
\left\|T_{n}^{-}(\delta, \cdot)\right\|_{p}=\left(\int_{-\delta}^{\delta}\left|T_{n}(\delta, t)\right|^{p} d t\right)^{1 / p} \leqslant M^{-}(2 \delta)^{1 / p} \leqslant C n^{3} \delta^{2+1 / p} .
$$

Теперь оценим $\left\|T_{n}^{-}(\delta, \cdot)\right\|_{p}$ снизу, для этого воспользуемся оценкой (2.3) леммы 2 . Так как $M^{-}<M^{+}$, имеем

$$
\begin{aligned}
\left\|T_{n}^{-}(\delta, \cdot)\right\|_{p} & \geqslant\left(C_{1} M^{-} n^{-2 /(2 p+1)}\left(M^{+}\right)^{-1 /(2 p+1)}\right)^{(2 p+1) /(2 p)} \\
& \geqslant C_{2}\left(n^{3} \delta^{2} n^{-2 /(2 p+1)} n^{-1 /(2 p+1)}\right)^{(2 p+1) /(2 p)}=C_{2} n^{3} \delta^{2+1 / p} .
\end{aligned}
$$

Лемма доказана.

ДоКАЗАТЕЛЬСТВо ТЕОРЕМЫ 1. ВоЗьмем

$$
\delta=\frac{n^{-3 p /(2 p+1)}}{2}=\frac{n^{-3 /(2+1 / p)}}{2},
$$

и, учитывая (3.13), (3.16), получаем, что существует константа $C>0$, не зависящая от $n$, такая, что

$$
\left\|T_{n}(\delta, \cdot)\right\|_{1, \infty} \geqslant C n^{3 /(2 p+1)}\left\|T_{n}(\delta, \cdot)\right\|_{1, p} \quad \forall p \in[1,+\infty] .
$$

Учитывая результаты предыдущего пункта, мы, тем самым, доказали теорему 1.

4. В этом пункте оценка (1.3) будет распространена на случай произвольных $p_{1}, p_{2}$, $q_{1}, q_{2} \in[1,+\infty]$ в задаче

$$
\sup _{\substack{t_{n} \in T_{n} \\ t_{n} \neq 0}} \frac{\left\|t_{n}\right\|_{q_{1}, q_{2}}}{\left\|t_{n}\right\|_{p_{1}, p_{2}}} .
$$

Пусть $t_{n} \in T_{n}$, как и в лемме 2 , рассмотрим два случая.

а) Пусть $M^{-}=M$. Тогда имеем

$$
M^{-} \leqslant e^{1 / e} n^{1 / p_{2}}\left\|t_{n}^{-}\right\|_{p_{2}}, \quad p_{2} \in[1,+\infty] .
$$

Пусть $p_{2}, q_{2} \in[1,+\infty]$ такие, что $q_{2} \geqslant p_{2}$. Тогда

$$
\begin{aligned}
\left\|t_{n}^{-}\right\|_{q_{2}} & =\left(\int_{0}^{2 \pi}\left|t_{n}^{-}(x)\right|^{q_{2}} d x\right)^{1 / q_{2}} \leqslant\left(M^{-}\right)^{1-p_{2} / q_{2}}\left\|t_{n}^{-}\right\|_{p_{2}}^{p_{2} / q_{2}} \\
& \leqslant e^{\left(1-p_{2} / q_{2}\right) / e} n^{1 / p_{2}-1 / q_{2}}\left\|t_{n}^{-}\right\|_{p_{2}} .
\end{aligned}
$$


При $q_{2} \leqslant p_{2}$ согласно неравенству Гёльдера

$$
\left\|t_{n}^{-}\right\|_{q_{2}} \leqslant C\left\|t_{n}^{-}\right\|_{p_{2}}
$$

Итак, в первом случае мы получили

$$
\left\|t_{n}^{-}\right\|_{q_{2}} \leqslant C n^{\left(1 / p_{2}-1 / q_{2}\right)+}\left\|t_{n}^{-}\right\|_{p_{2}} \leqslant C n^{\left(1 / p_{2}-1 / q_{2}\right)+}\left\|t_{n}\right\|_{p_{1}, p_{2}}, \quad p_{1}, p_{2} \in[1,+\infty] .
$$

b) Рассмотрим второй случай, когда $M^{-}<M^{+}$. Имеем

$$
M^{-} \leqslant e^{1 / e} n^{2 /\left(2 p_{2}+1\right)}\left\|t_{n}^{-}\right\|_{p_{2}}^{2 p_{2} /\left(2 p_{2}+1\right)}\left(M^{+}\right)^{1 /\left(2 p_{2}+1\right)}
$$

и, так как $M^{+}=M$, то опять по лемме 2 (случай а))

$$
M^{+} \leqslant e^{1 / e} n^{1 / p_{1}}\left\|t_{n}^{+}\right\|_{p_{1}}, \quad p_{1} \in[1,+\infty]
$$

откуда

$$
\begin{aligned}
M^{-} & \leqslant e^{1 / e} n^{2 /\left(2 p_{2}+1\right)}\left\|t_{n}^{-}\right\|_{p_{2}}^{2 p_{2} /\left(2 p_{2}+1\right)}\left(M^{+}\right)^{1 /\left(2 p_{2}+1\right)} \\
& \leqslant C_{1} n^{2 /\left(2 p_{2}+1\right)} n^{1 /\left(p_{1}\left(2 p_{2}+1\right)\right)}\left\|t_{n}^{-}\right\|_{p_{2}}^{2 p_{2} /\left(2 p_{2}+1\right)}\left\|t_{n}^{+}\right\|_{p_{1}}^{1 /\left(2 p_{2}+1\right)} .
\end{aligned}
$$

Отсюда по (2.2), полагая $a=\left\|t_{n}^{-}\right\|_{p_{2}}, b=\left\|t_{n}^{+}\right\|_{p_{1}}$, получаем

$$
M^{-} \leqslant C_{1} n^{\left(2+1 / p_{1}\right) /\left(2 p_{2}+1\right)}\left\|t_{n}\right\|_{p_{1}, p_{2}}
$$

Пусть $q_{2} \leqslant p_{2}$, тогда по неравенству Гёльдера $\left\|t_{n}^{-}\right\|_{q_{2}} \leqslant C_{2}\left\|t_{n}^{-}\right\|_{p_{2}}$. Пусть $q_{2} \geqslant p_{2}$, тогда

$$
\begin{aligned}
\left\|t_{n}^{-}\right\|_{q_{2}} & =\left(\int_{0}^{2 \pi}\left|t_{n}^{-}(x)\right|^{q_{2}} d x\right)^{1 / q_{2}}=\left(\int_{0}^{2 \pi}\left|t_{n}^{-}(x)\right|^{q_{2}-p_{2}}\left|t_{n}^{-}(x)\right|^{p_{2}} d x\right)^{1 / q_{2}} \\
& \leqslant\left(M^{-}\right)^{1-p_{2} / q_{2}}\left\|t_{n}^{-}\right\|_{p_{2}}^{p_{2} / q_{2}} \leqslant C_{1}^{1-p_{2} / q_{2}} n^{\frac{2+1 / p_{1}}{2+1 / p_{2}}\left(1 / p_{2}-1 / q_{2}\right)}\left\|t_{n}\right\|_{p_{1}, p_{2}} .
\end{aligned}
$$

Итак, во втором случае получили

$$
\left\|t_{n}^{-}\right\|_{q_{2}} \leqslant C n^{\frac{2+1 / p_{1}}{2+1 / p_{2}}\left(1 / p_{2}-1 / q_{2}\right)_{+}}\left\|t_{n}\right\|_{p_{1}, p_{2}} .
$$

Учитьвая оценки (4.1) и (4.2), окончательно получаем для любого $t_{n} \in T_{n}$

$$
\left\|t_{n}^{-}\right\|_{q_{2}} \leqslant C n^{\left(1 / p_{2}-1 / q_{2}\right)_{+} \max \left(1, \frac{2+1 / p_{1}}{2+1 / p_{2}}\right)}\left\|t_{n}\right\|_{p_{1}, p_{2}}, \quad p_{1}, p_{2}, q_{2} \in[1,+\infty] .
$$

Аналогично получаем

$$
\left\|t_{n}^{+}\right\|_{q_{1}} \leqslant C n^{\left(1 / p_{1}-1 / q_{1}\right)+\max \left(1, \frac{2+1 / p_{2}}{2+1 / p_{1}}\right)}\left\|t_{n}\right\|_{p_{1}, p_{2}}, \quad p_{1}, p_{2}, q_{2} \in[1,+\infty] .
$$

Мы доказали следующее утверждение. 
УТВЕРЖДЕНИЕ 2. Пусть $n \in \mathbb{N}, t_{n} \in T_{n}, p_{1}, p_{2}, q_{1}, q_{2} \in[1,+\infty]$. Тогда

$$
\left\|t_{n}\right\|_{q_{1}, q_{2}} \leqslant C n^{\psi\left(p_{1}, p_{2}, q_{1}, q_{2}\right)}\left\|t_{n}\right\|_{p_{1}, p_{2}},
$$

ฉде

$\psi\left(p_{1}, p_{2}, q_{1}, q_{2}\right)=\max \left(\left(\frac{1}{p_{1}}-\frac{1}{q_{1}}\right)_{+} \max \left(1, \frac{2+1 / p_{2}}{2+1 / p_{1}}\right),\left(\frac{1}{p_{2}}-\frac{1}{q_{2}}\right)_{+} \max \left(1, \frac{2+1 / p_{1}}{2+1 / p_{2}}\right)\right)$.

ДоКАЗАТЕЛЬСТво ТЕОРЕМЫ 2. В дополнение к неравенству (4.3) нужно доказать, что оно неулучшаемо. В случае $p_{1} \leqslant p_{2}$

$$
\psi\left(p_{1}, p_{2}, q_{1}, q_{2}\right)=\max \left(\left(\frac{1}{p_{1}}-\frac{1}{q_{1}}\right)_{+}, \frac{2+1 / p_{1}}{2+1 / p_{2}}\left(\frac{1}{p_{2}}-\frac{1}{q_{2}}\right)_{+}\right) .
$$

Если этот максимум достигается на $\left(1 / p_{1}-1 / q_{1}\right)_{+}$, то экстремальным полиномом будет $t_{n}=F_{n}(t)$, для которого

$$
\left\|F_{n}(\cdot)\right\|_{q_{1}, q_{2}}=\left\|F_{n}(\cdot)\right\|_{q_{1}} \asymp n^{1 / p_{1}-1 / q_{1}}\left\|F_{n}(\cdot)\right\|_{p_{1}, p_{2}} .
$$

Иначе (максимум достигается на $\left.\frac{2+1 / p_{1}}{2+1 / p_{2}}\left(1 / p_{2}-1 / q_{2}\right)_{+}\right)$экстремальным полиномом будет

$$
t_{n}=T_{n}(\delta, t) \quad \text { при } \delta=\frac{n^{-\frac{2+1 / p_{1}}{2+1 / p_{2}}}}{2} .
$$

Поскольку согласно оценкам (3.13) и (3.16) имеем

$$
\left\|T_{n}(\delta, \cdot)\right\|_{q_{1}, q_{2}} \asymp n^{\frac{2+1 / p_{1}}{2+1 / p_{2}}\left(1 / p_{2}-1 / q_{2}\right)}\left\|T_{n}(\delta, \cdot)\right\|_{p_{1}, p_{2}} .
$$

В случае $p_{1} \geqslant p_{2}$

$$
\psi\left(p_{1}, p_{2}, q_{1}, q_{2}\right)=\max \left(\frac{2+1 / p_{2}}{2+1 / p_{1}}\left(\frac{1}{p_{1}}-\frac{1}{q_{1}}\right)_{+},\left(\frac{1}{p_{2}}-\frac{1}{q_{2}}\right)_{+}\right),
$$

если этот максимум достигается на $\left(1 / p_{2}-1 / q_{2}\right)_{+}$, то экстремальным полиномом будет $t_{n}=-F_{n}(t)$, для которого

$$
\left\|-F_{n}(\cdot)\right\|_{q_{1}, q_{2}}=\left\|F_{n}(\cdot)\right\|_{q_{2}} \asymp n^{1 / p_{2}-1 / q_{2}}\left\|-F_{n}(\cdot)\right\|_{p_{1}, p_{2}} .
$$

Иначе (максимум достигается на $\left.\frac{2+1 / p_{2}}{2+1 / p_{1}}\left(1 / p_{1}-1 / q_{1}\right)_{+}\right)$экстремальным полиномом будет

$$
t_{n}=-T_{n}(\delta, t) \quad \text { при } \delta=\frac{n^{-\frac{2+1 / p_{2}}{2+1 / p_{1}}}}{2} .
$$

Так как согласно оценкам (3.13) и (3.16) имеем

$$
\left\|-T_{n}(\delta, \cdot)\right\|_{q_{1}, q_{2}} \asymp n^{\frac{2+1 / p_{2}}{2+1 / p_{1}}\left(1 / p_{1}-1 / q_{1}\right)}\left\|-T_{n}(\delta, \cdot)\right\|_{p_{1}, p_{2}} .
$$

Теорема 2 доказана.

Из соотношений $\|f\|_{p, p} \asymp\|f\|_{p} \forall p \in[1,+\infty]$ и теоремы 2 мы получаем 
СлеДСтвиЕ. Пусть $p, q \in[1,+\infty], n \in \mathbb{N}$. Тогда

$$
\sup _{\substack{t_{n} \in T_{n} \\ t_{n} \not 00}} \frac{\left\|t_{n}\right\|_{q}}{\left\|t_{n}\right\|_{p}} \asymp n^{(1 / p-1 / q)_{+}} .
$$

Таким образом, в качестве следствия из теоремы 2 мы получили оценку (1.1) с константой, не зависящей от $n$, и проверили ее неулучшаемость в смысле порядка.

Автор выражает искреннюю благодарность А.В. Рождественскому, С.Б. Стечкину, О. А. Тимошину, И. Г. Царькову за помошь при подготовке работы. Автор также признателен В.В. Арестову, которьй прочитал рукопись и сделал ряд ценных замечаний. Работа выполнена под руководством С. Б. Стечкина.

Московский государственный университет

Поступило

им. М.В. Ломоносова

18.08 .95

\section{СПИСОК ЦИТИРОВАННОЙ ЛИТЕРАТУРЫ}

[1] Никольский С. М. Приближение функций многих переменных и теоремы вложения. М.: Наука, 1977.

[2] Jackson D. Certain problem of closest approximation // Bull. Amer. Math. Soc. 1933. V. 39. P. 889-906.

[3] Стечкин С. Б. Обобщение некоторых неравенств С.Н. Бернштейна // Докл. АН СССР. 1948. T. 60. №9. C. 1511-1514.

[4] Дзядык В.К. Введение в теорию равномерного приближения функций полиномами. М.: Наука, 1977.

[5] Тиман А.Ф. Теория приближений функций действительного переменного. М.: Физматгиз, 1960. 